\title{
Low-Complexity Scheduling and Power Adaptation for Coordinated Cloud-Radio Access Networks
}

\author{
Ahmed Douik, Student Member, IEEE, Hayssam Dahrouj, Senior Member, IEEE, \\ Tareq Y. Al-Naffouri, Member, IEEE, and Mohamed-Slim Alouini, Fellow, IEEE
}

\begin{abstract}
In practical wireless systems, the successful implementation of resource allocation techniques strongly depends on the algorithmic complexity. Consider a cloud-radio access network (CRAN), where the central cloud is responsible for scheduling devices to the frames' radio resources blocks (RRBs) of the single-antenna base-stations (BSs), adjusting the transmit power levels, and for synchronizing the transmit frames across the connected BSs. Previous studies show that the jointly coordinated scheduling and power control problem in the considered CRAN can be solved using an approach that scales exponentially with the number of BSs, devices, and RRBs, which makes the practical implementation infeasible for reasonably sized networks. This paper instead proposes a low-complexity solution to the problem, under the constraints that each device cannot be served by more than one BS but can be served by multiple RRBs within each BS frame, and under the practical assumption that the channel is constant during the duration of each frame. The paper utilizes graph-theoretical based techniques and shows that constructing a single power control graph is sufficient to obtain the optimal solution with a complexity that is independent of the number of RRBs. Simulation results reveal the optimality of the proposed solution for slow-varying channels, and show that the solution performs near-optimal for highly correlated channels.
\end{abstract}

Index Terms-Joint scheduling and power adaptation, slowvarying channels, complexity reduction.

\section{INTRODUCTION}

The ever increasing demand for bandwidth-hungry services is pushing wireless networks toward additional spectrum reuse, which causes exacerbated wireless interference levels. A promising large-scale interference management technique is to connect the different base-stations (BSs) to a central unit, i.e., a cloud, which is responsible for the joint resource allocation, e.g., jointly encoding (decoding) the messages using downlink (uplink) coordinated resource allocation techniques. Thanks to its ability to control BSs transmissions, the CRAN infrastructure is a suitable and cost-efficient solution for next generation wireless systems (5G).

In practical CRAN deployment, the cloud may be geographically far from the BSs, and so joint signal processing may not always be feasible as it requires high capacity backhaul links so as to connect all BSs to the central cloud. An alternative coordination approach is only to share the scheduling policy and the power levels of each RRB among the BSs, through centralized computations at the cloud [1]. This paper

Hayssam Dahrouj would like to thank Effat University in Jeddah, Saudi Arabia, for funding the research reported in this paper through the Research and Consultancy Institute

Ahmed Douik is with the Department of Electrical Engineering, California Institute of Technology, Pasadena, CA 91125 USA (e-mail: ahmed.douik@caltech.edu).

Hayssam Dahrouj is with the Department of Electrical and Computer Engineering, Effat University, Jeddah 22332, Saudi Arabia (e-mail: hayssam.dahrouj@gmail.com).

T. Y. Al-Naffouri and M.-S. Alouini are with the Division of Computer, Electrical and Mathematical Sciences and Engineering, King Abdullah University of Science and Technology, Thuwal 23955-6900, Saudi Arabia (e-mail: \{tareq.alnaffouri,slim.alouini\}@kaust.edu.sa). inscribes itself along such direction, as it considers a CRAN architecture where the central cloud is only responsible for jointly scheduling users to the frames' RRBs of single-antenna BSs and adjusting their transmit power levels, as well as for synchronizing the transmit frames across the connected BSs. Such coordination is practically feasible, as it only requires low-capacity backhaul links.

Network resource optimization in CRANs can be divided into two main categories namely the energy consumption reduction under quality of service constraints, e.g., [2], [3] and the throughput maximizatin under power budget constraints. The coordinated scheduling and power control problem in this paper focuses on finding the optimal assignment of users to the RRBs of the BSs and the power level of each RRB by maximizing the network-wide weighted sum rate, under the constraints that each device cannot be served by more than one BS but can be served by multiple RRBs within each BS frame. The considered joint optimization problem is mathematically challenging. To addresses such difficulty, a large body of literature, e.g., [4], considers a modular approach to solve the problem, wherein the scheduling is updated in an inner loop for a fixed power level, and the power is updated in an outer loop for a fixed schedule. However, such a modular approach is sub-optimal and may not fully exploit the network potential.

In the context of a multi-cell OFDMA networks with negligible intercell interference, the authors in [5] propose a joint optimization algorithm. While reference [6] presents a branch-and-bound algorithm that is guaranteed to converge to a stationary point of the overall joint scheduling and power control problem, the optimal solution is achieved in [1] using a graph theoretical approach. Reference [1] solves the problem by introducing the joint scheduling and power control graph wherein each vertex represents a combination of user, BS, RRB, and power level. The problem is reformulated as a maximum weight clique problem over the designed graph. Reference [1] particularly shows that the joint approach provides significant performance gain as compared to the conventional iterative schemes. However, the proposed solution scales exponentially in the number of users, BSs, and RRBs, which may not be feasible for a moderately large network.

The main contribution of this letter is to propose a lowcomplexity, yet optimal, solution to the joint scheduling and power control problem, under the practical observation that the channel is typically constant within each frame RRBs. The paper shows that the optimal solution can be reached by constructing a single local power control graph. Such simplification primarily impacts the complexity of the solution as it becomes independent of the number of RRBs in the network. Simulation results reveal the optimality of the proposed solution for slow-varying channels, and show that the solution performs near-optimal for highly correlated channels. 


\section{System Model and Problem Formulation}

\section{A. System Model}

Consider the downlink of a cloud radio-access network consisting of $B$ single-antenna BSs. The transmit frame of each BS is composed of $R$ orthogonal radio resource blocks. The network comprises $B R$ RRBs in total, each with its own power level. All BSs are connected to a central unit, i.e., the cloud, which is responsible for synchronizing the transmit frames across all BSs, scheduling the $U$ users to each frame RRB, and determining the power level of each RRB. The set of BSs, RRBs, and users are denoted by the calligraphic letters $\mathcal{B}, \mathcal{R}$, and $\mathcal{U}$, respectively. Thanks of the cloud synchronization ability, the signal-to-interference plus noise-ratio (SINR) of the $u$-th user, scheduled to the $b$-th $\mathrm{BS}$ at the $r$-th RRB is given by $\operatorname{SINR}_{u b r}(\mathbf{P})=\frac{P_{b r}\left|h_{u b r}\right|^{2}}{\sigma^{2}+\sum_{b^{\prime} \neq b} P_{b^{\prime} r}\left|h_{u b^{\prime} r}\right|^{2}}$, where $h_{u b r}$ is the corresponding channel between the $u$-th device and the $r$-th RRB at the $b$-th BS, and $P_{b r}$ is the power level used by the $b$-th BS at the $r$-th RRB bounded by the maximal power the base-station can deliver, i.e., $P_{b r} \leq P_{b}^{\max }$. The paper assumes that the channels are slow-varying. In other terms, if the RRB represents a frequency block, then the channel is non-frequency selective. If the RRB represents a time block, it assumes that the duration of the entire frame is within the channel coherence time. Hence, the channel remains constant across each frame RRBs within the same BS, i.e., $h_{u b r}=h_{u b}, \forall r \in \mathcal{R}, \forall b \in \mathcal{B}, \forall u \in \mathcal{U}$. The additive white Gaussian noise variance is denoted by $\sigma^{2}$.

\section{B. Problem Formulation}

The joint coordinated scheduling and power control in the context of this paper is the problem of assigning users to RRBs, under the constraint that each user can be connected to at most a single BS, and that each RRB needs to be allocated to one and only one user. Introduce the binary variable $X_{u b r}$ that is 1 if the $u$-th user is assigned to the $r$-th RRB in the $b$-th BS frame. Similarly, let $Y_{u b}$ be a binary variable that is 1 if user $u$ is mapped to the $b$ th $\mathrm{BS}$, and zero otherwise. Finally, let $\pi_{u b r}=a_{u b} \log _{2}\left(1+\operatorname{SINR}_{u b r}(\mathbf{P})\right)$ be the weighted rate of the $u$-th user when connected to the $r$-th RRB in the $b$-th BS, where $a_{u b}$ is a positive constant that is typically introduced for fairness and load balancing purposes. Updating $a_{u b}$ typically happens in an outer loop, which falls outside the scope of the current paper. The network-wide weighted sumrate optimization problem considered in this paper can then be formulated as follows:

$$
\begin{array}{ll}
\max & \sum_{u, b, r} \pi_{u b r} X_{u b r} \\
\text { s.t. } & Y_{u b}=\min \left(\sum_{r} X_{u b r}, 1\right), \forall(u, b) \in \mathcal{U} \times \mathcal{B}, \\
& \sum_{b} Y_{u b} \leq 1, \quad \forall u \in \mathcal{U}, \\
& \sum_{u} X_{u b r}=1, \quad \forall(b, r) \in \mathcal{B} \times \mathcal{R}, \\
& 0 \leq P_{b r} \leq P_{b}^{\max }, \forall(b, r) \in \mathcal{B} \times \mathcal{R}, \\
& X_{u b r}, Y_{u b} \in\{0,1\}, \forall(u, b, r) \in \mathcal{U} \times \mathcal{B} \times \mathcal{R},
\end{array}
$$

where the optimization is over the discrete variables $X_{u b r}$ and $Y_{u b}$, the continuous variables $P_{b r}$, where constraints (1b) and (1c) state that a device can be assigned to at most a single BS, where constraint (1d) states that each RRB needs to be assigned, and where (1d) is the maximum power constraint on every RRB.

Problem (1) is a mixed continuous and discrete optimization problem. The optimal solution to such a problem requires constructing $R$ local power graphs and solving the maximum weight clique problem over the union of these graphs [1]. Such union of graphs, known as the joint scheduling and power control graph, has a size that is up to $\left({ }^{U} P_{B}\right) \cdot R$, where ${ }^{n} P_{r}=$ $n ! /(n-r) !$ is the number of possible permutations. Such a solution, albeit optimal, is of high computational complexity, even for reasonably-sized graphs. The next section shows that the solution can indeed be achieved with a noticeable complexity reduction, when the channel is fixed across the RRBs of each frame, as it is the case in the current paper.

\section{Proposed SOlution}

The first part of this section presents the joint scheduling and power control graph used to find the optimal solution of problem (1). The second part then exploits the fact that the channel is fixed across the RRBs of each frame and proposes a complexity-efficient solution to the problem. The second part particularly shows that for slow-varying channels, the optimal solution can be achieved by constructing a single local power control graph. Finally, the section characterizes the complexity of the proposed solution and concludes its computation efficiency.

\section{A. Power-Control and Scheduling Graph}

The joint scheduling and power control graph consists of the union of $R$ local power control graphs. This section first describes the construction of a local power control graph. Afterward, it gives the connectivity condition between the various graphs to form the joint scheduling and power control graph.

Let $\mathcal{A}=\mathcal{U} \times \mathcal{B} \times \mathcal{R}$ be the set of all possible associations between users, BSs, and RRBs. Define the function $\varphi_{u}$ as the mapping function from the set $\mathcal{A}$ to the set of users $\mathcal{U}$, i.e., $\varphi_{u}(y)=u, \forall y=(u, b, r, p) \in \mathcal{A}$. Similarly, define $\varphi_{b}$ and $\varphi_{r}$ as the mapping functions from $\mathcal{A}$ to the set of BSs and RRBs, respectively. Let $\mathcal{F}_{r}$ be the set of the feasible associations for RRBs indexed by $r$ defined by:

$$
\begin{aligned}
\mathcal{F}_{r}= & \left\{\mathbf{S}_{r} \in\left\{\mathcal{U}, \mathcal{B}, r, \mathcal{P}_{r}\right\}^{B}\right. \text { such that } \\
& \left.\varphi_{u}(s) \neq \varphi_{u}\left(s^{\prime}\right) \text { and } \varphi_{b}(s) \neq \varphi_{b}\left(s^{\prime}\right), \forall s \neq s^{\prime} \in \mathbf{S}_{r}\right\},
\end{aligned}
$$

where the set $\mathcal{P}_{r}$ is obtained by solving the following power allocation problem for each combination of users and BS represented by the association $\mathbf{S}_{r}$ for a fixed RRB $r$ :

$$
\max _{p_{b r}} \sum_{b \in \mathcal{B}} \pi_{u b r} \text { subject to } 0 \leq p_{b r} \leq P_{b}^{\max }, \forall b \in \mathcal{B} .
$$

The above power allocation problem (2) is a well-known problem that can be solved optimally [7]. For the $r$-th RRB, the local power control graph $\mathcal{G}_{r}\left(\mathcal{V}_{r}, \mathcal{E}_{r}\right)$ is constructed by creating a vertex $v_{r} \in \mathcal{V}_{r}$ for each possible association $\mathbf{S}_{r} \in$ 
$\mathcal{F}_{r}$. This graph does not contain any connection, i.e., $\mathcal{E}_{r}=\varnothing$. The weight of each vertex is the weighted sum rate of the represented association. The weight of vertex $v_{r}$ associated with the schedule $\mathbf{S}_{r}$ is $w\left(v_{r}\right)=\sum_{s \in \mathbf{S}_{r}} \pi\left(s, \mathbf{S}_{r}\right)$.

The joint coordination and power control graph is then constructed by taking all vertices from all local power control graphs. Two vertices $v_{r} \in \mathcal{G}_{r}$ and $v_{r^{\prime}} \in \mathcal{G}_{r^{\prime}}$, representing the associations $\mathbf{S}_{r}$ and $\mathbf{S}_{r^{\prime}}$, are connected if:

$$
\begin{aligned}
& \delta\left(\varphi_{u}\left(s_{r}\right)-\varphi_{u}\left(s_{r^{\prime}}\right)\right) \delta\left(\varphi_{b}\left(s_{r}\right)-\varphi_{b}\left(s_{r^{\prime}}\right)\right) \\
& \quad=\delta\left(\varphi_{u}\left(s_{r}\right)-\varphi_{u}\left(s_{r^{\prime}}\right)\right), \forall\left(s_{r_{1}}, s_{r^{\prime}}\right) \in \mathbf{S}_{r} \times \mathbf{S}_{r^{\prime}}
\end{aligned}
$$

The optimal solution of the coordinated scheduling problem (1) can then be found by leveraging the method presented in [1] for our problem (1). Such solution is the maximum-weight clique of degree $R$ in the joint scheduling and power control graph.

\section{B. Low-Complexity Scheduling and Power Control}

As shown in the previous section, the complexity of the optimal solution scales exponentially with the number of the available resources, which is typically large. Such solution, however, does not take into account the fact that $h_{u b r}=$ $h_{u b}, \forall r \in \mathcal{R}, \forall b \in \mathcal{B}, \forall u \in \mathcal{U}$. The following theorem introduces the solution of the joint coordinated scheduling and power control problem (1) under the current paper scenario.

Theorem 1. Let $\mathcal{G}_{r}\left(\mathcal{V}_{r}, \mathcal{E}_{r}\right)$ be the local power control graph for an arbitrary $R R B r \in \mathcal{R}$, and let $v=\left\{s_{1}, \cdots, s_{B}\right\}$ be the vertex with maximum weight. The optimal schedule is the one that schedules user $\varphi_{u}\left(s_{i}\right)$ to all RRBs in BS $\varphi_{b}\left(s_{i}\right), 1 \leq$ $i \leq B$ and the generated sum rate is given by $w(v) R$ where $w(v)$ is the weight of vertex $v$.

Proof: Let $\mathcal{C}^{*}=\left\{v_{1}, \cdots, v_{R}\right\}$ be the maximum weight clique of size $R$ in the joint coordination and power control graph, and let $\mathbf{C}$ be the set of all cliques of size $R$. First note from Lemma 3 in [1] that each vertex of the maximum weight clique belongs to a distinct local power control graph. Without loss of generality, assume that $v_{r} \in \mathcal{G}_{r}$. The maximum weight clique problem can be written as:

$$
\begin{gathered}
w\left(\mathcal{C}^{*}\right)=\max _{\substack{v_{r} \in \mathcal{G}_{r} \\
\mathcal{C} \in \mathbf{C}}} w(\mathcal{C})=\max _{\substack{v_{r} \in \mathcal{G}_{r} \\
\mathcal{C} \in \mathbf{C}}} \sum_{r \in \mathcal{R}} w\left(v_{r}\right) \leq \max _{v_{r} \in \mathcal{G}_{r}} \sum_{r \in \mathcal{R}} w\left(v_{r}\right) \\
\leq \sum_{r \in \mathcal{R}} \max _{v_{r} \in \mathcal{G}_{r}} w\left(v_{r}\right)=\sum_{r \in \mathcal{R}} w\left(v_{r}^{*}\right)
\end{gathered}
$$

where $v_{r}^{*}$ is the vertex with maximum weight in the local power control graph $\mathcal{G}^{r}, \forall r \in \mathcal{R}$.

To prove the theorem, it is now sufficient to prove that the set $\left\{v_{r}^{*}\right\}_{r \in \mathcal{R}}$ is a clique in the joint coordination and power control graph and that $w\left(v_{r}^{*}\right)=w^{*}, \forall r \in \mathcal{R}$.

Recall that the weight of each vertex in the local power control graph depends solely on the SINR of the scheduling represented by that vertex. Therefore, showing that the same scheduling for different RRBs has the same SINR is sufficient to conclude that the corresponding vertices have the same weight. In other words, it is sufficient to show that the SINR can be written as $\operatorname{SINR}_{u b r}(\mathbf{P})=\operatorname{SINR}_{u b}(\mathbf{P})$. In particular, the vertex with the highest weight represents the same scheduling at the different RRBs. This holds because of the assumption that $h_{u b r}=h_{u b}, \forall r \in \mathcal{R}, \forall b \in \mathcal{B}, \forall u \in \mathcal{U}$. In fact, for the same scheduling, the power optimization problem for RRBs $r$ and $r^{\prime}$ are equivalent, i.e., they can be written as the following optimization problem over dummy positive variables $z_{b}$ :

$\max _{z_{b}} \sum_{b \in \mathcal{B}} a_{u b} \log _{2}\left(1+\frac{z_{b}\left|h_{u b}\right|^{2}}{\sigma^{2}+\sum_{b^{\prime} \neq b} z_{b^{\prime}}\left|h_{u b^{\prime}}\right|^{2}}\right)$, s.t. $z_{b} \leq P_{b}^{\max }$

Let $v_{r_{1}}^{*}=\left\{s_{1}^{r_{1}}, \cdots, s_{B}^{r_{1}}\right\}$ and $v_{r_{2}}^{*}=\left\{s_{1}^{r_{2}}, \cdots, s_{B}^{r_{2}}\right\}, r_{1} \neq$ $r_{2} \in \mathcal{R}$ be the two vertices with maximum weight in $\mathcal{G}_{r_{1}}$ and $\mathcal{G}_{r_{2}}$, respectively. From the above analysis, these two vertices represent the same scheduling of users to BSs for RRBs $r_{1}$ and $r_{2}$. Hence if $\varphi_{u}\left(s_{i}^{r_{1}}\right)=\varphi_{u}\left(s_{j}^{r_{2}}\right)$ for some $i, j$ then $\varphi_{u}\left(s_{i}^{r_{1}}\right)=$ $\varphi_{u}\left(s_{j}^{r_{2}}\right)$. In other words, we have

$$
\begin{aligned}
& \delta\left(\varphi_{u}\left(s_{i}^{r_{1}}\right)-\varphi_{u}\left(s_{j}^{r_{2}}\right)\right) \delta\left(\varphi_{b}\left(s_{i}^{r_{1}}\right)-\varphi_{b}\left(s_{j}^{r_{2}}\right)\right) \\
&=\delta\left(\varphi_{u}\left(s_{i}^{r_{1}}\right)-\varphi_{u}\left(s_{j}^{r_{2}}\right)\right), 1 \leq i, j \leq B
\end{aligned}
$$

Therefore, each pair of vertices $v_{r_{1}}$ and $v_{r_{2}}$ verify the connectivity condition, which concludes that $\left\{v_{r}^{*}\right\}_{r \in \mathcal{R}}$ is a clique in the joint coordination and power control graph.

The proof of Theorem 1 further shows that the proposed schedule is always a feasible one, regardless of the channel variations. The proposed algorithm can, therefore, be used for highly correlated channel although it no longer guarantees optimality, as the simulations section illustrates next.

\section{Complexity Analysis}

This subsection characterizes the computation complexity of the proposed solution and compares it with the complexity of state of the art algorithms. To that end, define $C_{p}$ as the complexity of solving the power allocation problem (2) and let $1<\alpha \leq 2$ be the complexity constant for solving the maximum weight clique problem. Furthermore, let $T$ be the number of iterations between the scheduling and power control problems.

As shown in Theorem 1, the optimal solution requires constructing a local power control graph and solving the power allocation for each of its vertices. The graph contains ${ }^{U} P_{B}$ nodes, and so the computational complexity of the proposed algorithm is $C_{p}\left({ }^{U} P_{B}\right)$, which scales exponentially with respect to the number of base station and users, but not with respect to the number of resource blocks. The optimal solution proposed in [1] requires the construction of $R$ local graphs and solving the maximum weight clique in the union of these graphs, which produces a total complexity of $C_{p} R\left({ }^{U} P_{B}\right)+\alpha^{R\left({ }^{U} P_{B}\right)}$. The scheduling steps require solving a maximum weight clique in a graph with $U B R$ vertices, which yields a complexity of $\alpha^{U B R}$. Likewise, the iterative method solves the power allocation and scheduling independently $T$ times, which accounts for a total complexity of $T\left(C_{p}+\alpha^{U B R}\right)$.

\section{Simulation Results}

This section evaluates the performance of the proposed algorithm in the downlink of a cloud-radio access network composed of $3 \mathrm{BSs}$. The cell size is $500 \mathrm{~m}$, and the bandwidth of operation is $10 \mathrm{MHz}$. The noise and maximum power are set to $\sigma^{2}=-168.60 \mathrm{dBm} / \mathrm{Hz}$ and $P^{\max }=-42.60 \mathrm{dBm} / \mathrm{Hz}$. The channels gain $h_{u b r}$ is generated according to the following model: $h_{u b r}=(1-\lambda) h_{u b}+\lambda H_{u b r}$, where $h_{u b}$ and $H_{u b r}$ 


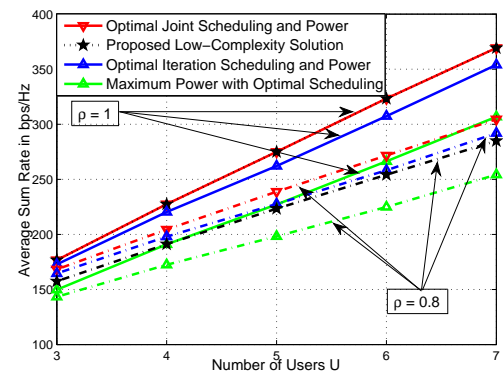

Fig. 1. Sum-rate in bps $/ \mathrm{Hz}$ versus the number of users $U$ for a network with fully (solid lines) and higly (dashed lines) correlated channels.

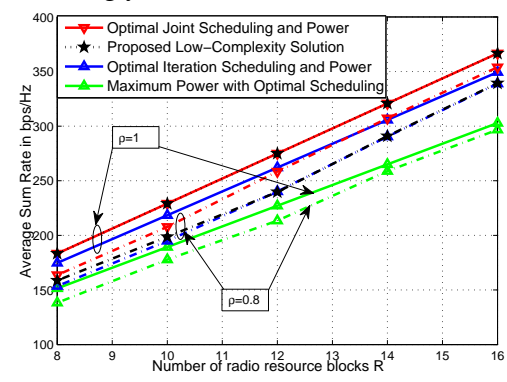

Fig. 2. Sum-rate in bps/Hz versus the number of RRBs $R$ for a network with fully (solid lines) and higly (dashed lines) correlated channels.

follow the SUI-3 terrain type B channel model. While $h_{u b}$ is common to all RRBs within the same BS frame, $H_{u b r}$ is induced to characterize the performance as a function of the channel correlation variations across the RRS. For example, $\lambda=1$ illustrates the case of same channel across all RRBs. $\lambda=0$ illustrates the case of different channels across different RRBs.

The correlation $\rho$ between a couple of channels is given by $\rho=\frac{\mathbb{E}\left[h_{u b r} h_{u b r^{\prime}}\right]}{\sigma\left(h_{u b r}\right) \sigma\left(h_{u b r^{\prime}}\right)}=\left(1+\left(\frac{\lambda}{1-\lambda}\right)^{2}\right)^{-1}$. Figure 1 and Figure 2 plot the sum-rate in bps/Hz versus the number of users and the number of RRBs in the network for a network with 12 RRBs per BS and 5 users, respectively. The figures consider the correlation cases of $\rho=1$ and $\rho=0.8$, which reflect the cases of perfectly and highly correlated channel gains, respectively. For perfectly correlated channels. i.e., same channel across all RRBs, the proposed low-complexity algorithm achieves the optimal solution of the joint scheduling and power control problem for all network configurations, as expected due to Theorem 1 result. Figure 1 further shows that for highly correlated channel gains, i.e., $\rho=0.8$, the proposed algorithm, which is suboptimal in this case, is not far off from the global optimal solution. Figure 2 particularly shows that, for both cases, the proposed algorithm outperforms the iterative classical approach where the scheduling is updated in an inner loop for a fixed power level, and the power is updated in an outer loop for a fixed schedule. The proposed algorithm also outperforms the scheduling solution which uses the maximum allowable power.

To evaluate the complexity of the proposed solution as a function of the algorithmic running time, Table IV summarizes the performance of the four solutions for different values of the channel correlation $\rho$. The considered network has 5 users, 3 BS, and 12 RRBs. The iterative algorithm iterates between the scheduling and power control problem 10 times before
TABLE I

PERFormance AND RUNNING TIME OF THE DiffERENT Algorithms

\begin{tabular}{|c|c|c|c|c|}
\hline Solution & $\rho=1$ & $\rho=0.9$ & $\rho=0.8$ & Time $(\mathrm{s})$ \\
\hline Optimal & 274.944 & 252.885 & 238.767 & 859.497 \\
\hline Proposed & 274.944 & 241.259 & 223.362 & 0.967 \\
\hline Iterative & 262.054 & 234.551 & 227.573 & 17.744 \\
\hline Max Power & 227.113 & 204.551 & 198.208 & 0.239 \\
\hline
\end{tabular}

outputting the solution. The simulations are carried in Matlab on a Windows 10 laptop $2.4 \mathrm{GHz}$ Intel Core i7 processor and $8 \mathrm{~GB} 1600 \mathrm{MHz}$ DDR3 RAM. It can clearly be seen from the table that the proposed scheme outperforms the other solutions for highly dependent channels, both from complexity and performance perspectives. The proposed algorithm offers lower computational complexity as compared to the optimal solution presented in [1]. It also outperforms the classical iterative algorithm.

\section{CONClusion}

This paper proposes a low-complexity solution to the joint coordination and power control in cloud-radio access networks with slow-varying channels. Previous studies show that the jointly coordinated scheduling and power control problem in the considered CRAN can be solved using an approach that scales exponentially with the number of BSs, devices, and RRBs, which makes the practical implementation infeasible for reasonably sized networks. The paper shows instead that the optimal solution can be obtained with a complexity that is independent from the number of available radio resource blocks. Such approach requires the construction of a single power control graph and selecting the vertex with the maximum weight. The suggested algorithm is, therefore, suitable to be implemented in large scale networks. Simulation results reveal the optimality of the proposed solution for slow-varying channels, and show that the solution performs near-optimal for highly correlated channels.

\section{REFERENCES}

[1] A. Douik, H. Dahrouj, T. Y. Al-Naffouri, and M. S. Alouini, "Coordinated scheduling and power control in cloud-radio access networks," IEEE Transactions on Wireless Communications, vol. 15, no. 4, pp. 2523-2536, April 2016.

[2] D. W. K. Ng, E. S. Lo, and R. Schober, "Energy-efficient resource allocation in multi-cell OFDMA systems with limited backhaul capacity," IEEE Transactions on Wireless Communications, vol. 11, no. 10, pp. 3618-3631, October 2012.

[3] Q. Wu, W. Chen, M. Tao, J. Li, H. Tang, and J. Wu, "Resource allocation for joint transmitter and receiver energy efficiency maximization in downlink OFDMA systems," IEEE Transactions on Communications, vol. 63, no. 2, pp. 416-430, Feb 2015.

[4] J.-W. Lee, R. Mazumdar, and N. Shroff, "Joint resource allocation and base-station assignment for the downlink in CDMA networks," IEEE/ACM Transactions on Networking, vol. 14, no. 1, pp. 1-14, Feb 2006.

[5] J. Huang, V. Subramanian, R. Agrawal, and R. Berry, "Joint scheduling and resource allocation in uplink OFDM systems for broadband wireless access networks," IEEE Journal on Selected Areas in Communications, vol. 27, no. 2, pp. 226-234, February 2009.

[6] L. Yu, E. Karipidis, and E. Larsson, "Coordinated scheduling and beamforming for multicell spectrum sharing networks using branch and bound," in Proc. of the 20th European Signal Processing Conference (EUSIPCO' 2012), Bucharest, Romania, Aug 2012, pp. 819-823.

[7] L. P. Qian, Y. Zhang, and J. Huang, "Mapel: Achieving global optimality for a non-convex wireless power control problem," IEEE Transactions on Wireless Communications, vol. 8, no. 3, pp. 1553-1563, March 2009. 